\title{
PROTECTIVE EFFECT OF PHYLLANTHUS EMBLICA EXTRACT PREVENT CONTRAST-INDUCED ACUTE KIDNEY INJURY IN RATS
}

\author{
SUPRANEE KONGKHAM ${ }^{1 *}$, ADIS TASANARONG ${ }^{2}$, ARUNPORN ITHARAT ${ }^{3}$ \\ ${ }^{1}$ Department of Preclinical Sciences, Division of Biochemistry, Faculty of Medicine, Thammasat University (Rangsit Campus), \\ Pathumthani, Thailand. 'Department of Medicine, Nephrology Unit, Faculty of Medicine, Thammasat University (Rangsit Campus), \\ Pathumthani, Thailand. ${ }^{3}$ Division of Applied Thai Traditional Medicine, Faculty of Medicine, Thammasat University (Rangsit Campus), \\ Pathumthani, Thailand. Email: ksuprane@tu.ac.th
}

Received: 23 July 2019, Revised and Accepted: 24 August 2019

\section{ABSTRACT}

Objective: The objective of the study was to investigate the anti-apoptosis effect of the extract from Phyllanthus emblica (PE) for the prevention of contrast-induced acute kidney injury (CI-AKI).

Methods: Male Sprague Dawley rats were given saline (control) or PE extracts (500 mg/kg/day) for 5 days before the induction of CI-AKI. Renal tissues were collected for an evaluation of gene expression and immunohistochemistry (IHC). To indicate anti-apoptotic effect, the expression levels of Bax, Bcl-2, and caspase in kidney were also determined, using real-time polymerase chain reaction (RT-PCR) and Western blot analysis.

Results: In the CI-AKI group, RT-PCR and Western blot analysis revealed that the expression levels of Bax and cleaved-caspase-3 were upregulated in the CI-AKI group, whereas the expression of Bcl-2 was downregulated. However, the pre-treatment with PE increased Bcl-2 expression. Moreover, decreased cleaved-caspases-3 activity was also detected using IHC

Conclusion: These findings suggested that pretreatment with PE extract provided the anti-apoptotic effect against CI-AKI in the rat model.

Keywords: Anti-apoptosis, Contrast-induced acute kidney injury, Phyllanthus emblica.

(c) 2019 The Authors. Published by Innovare Academic Sciences Pvt Ltd. This is an open access article under the CC BY license (http://creativecommons. org/licenses/by/4. 0/) DOI: http://dx.doi.org/10.22159/ajpcr.2019.v12i10.31755

\section{INTRODUCTION}

Contrast-induced acute kidney injury (CI-AKI), a complication resulted from the administration of iodinated intravascular contrast medium, is the third most common cause of hospital-acquired AKI [1,2]. CI-AKI is defined as an impairment of renal function $48-72 \mathrm{~h}$ after receiving the contrast medium with an absolute increase of serum creatinine by $0.5 \mathrm{mg} / \mathrm{dl}$ or more or a relative increase by $25 \%$ or more in the serum creatinine level from baseline. Although the exact incidence of CI-AKI remains unclear, the incidence in a high-risk population (such as patients with chronic renal impairment and/or diabetes mellitus) has increased up to $25 \%$, resulting in a significant rise of morbidity and mortality rate [2-5]. The pathogenesis of CI-AKI is due to the ability of the contrast agents that could induce renal vasoconstriction causing a decrease in renal blood flow and hypoxic milieu. In hypoxic tissue, the formation of reactive oxygen species (ROS) is increased, and the ROS that exceeds the cellular scavenging capacities can subsequently damage the surrounding cells and tissues [6]. The contrast media (CM) also have a direct toxic effect on tubular cells by directly inducing cellular apoptosis. Oxidative damage and apoptosis altogether contribute to the pathogenesis of CI-AKI. Thus, any intervention that can prevent apoptosis is important for improving nephropathy $[7,8]$.

Although there are many CI-AKI preventative strategies which have been tested in various experimental studies and clinical trials, hydration with isotonic saline has been recommended as a mainstay of CI-AKI prevention [9]. Additional antioxidants agents such as $\mathrm{N}$-acetylcysteine, ascorbic acid, Vitamin E, and other agents including statin and fenoldopam have been evaluated, but the prophylactic effects of these agents are still questionable, and they are not yet included in the recommendation for CI-AKI prevention $[4,10,11]$.
Phyllanthus emblica (PE) Linn. (synonym. Emblica officinalis Gaertn.), a member of the Euphorbiaceae family, is native to the South and Southeast Asia. In Thailand, it is commonly known as Makhampom. Its fruit serves as a rich source of Vitamin C, gallic acid, minerals, and amino acid [12-14]. It also contains mixture of phenolic compounds (such as flavonoids, tannins, terpenoids, and alkaloids) which have potent various biological activities including anticancer [15-17], radioprotection [17], and antiinflammatory [18]. Its antioxidant property has been shown in several injury-protection models of various organs including brain, heart, liver, kidney, and stomach [13,19-21]. Recently, PE has been reported that its antioxidant property accounts for the preventive effect in CI-AKI [22]. However, its anti-apoptotic effect of PE extract on CI-AKI has not been studied yet. Therefore, the main purpose of this study was to investigate whether the PE extract was able to attenuate apoptosis and to prevent CIAKI in rat models.

\section{METHODS}

\section{Sample preparations}

In the present study, all samples were taken from the previous work [22]. Therefore, the preventive effect of PE against CI-AKI has also confirmed by renal function test and histopathology examination. The kidney samples were taken only three groups including Group 1, control rat administrated with $1 \mathrm{ml}$ of normal saline; Group 2, rat administrated with $\mathrm{CM}$; ad Group 3 rats were orally administrated with PE $(500 \mathrm{mg} / \mathrm{kg} /$ day $)$ for 5 days before receiving CM on the following day. For protein study, small sections of the kidney tissues were frozen in liquid nitrogen and stored at $-70^{\circ} \mathrm{C}$ before being analyzed by Western blot analysis. Separated sections were fixed in RNAlater Stabilization Solution (Ambion, Inc.) for gene expression studies using real-time polymerase chain reaction (RTPCR). 
Immunohistochemistry (IHC) staining of cleaved caspase-3 in kidney tissue

Paraffin-embedded tissues were cut into $4 \mu \mathrm{m}$ thick slices for IHC. The sections were deparaffinized and rehydrated by passing through xylene, series of ethanol, and water. Antigen retrieval was performed by boiling the sections in sodium citrate buffer for 10 min and then steadily cooling at room temperature. Subsequently, the sections were blocked using $3.0 \%$ hydrogen peroxide in methanol for $15 \mathrm{~min}$ to inhibit endogenous peroxidase activity. Following washing in phosphate-buffered saline, the sections were incubated overnight at $4^{\circ} \mathrm{C}$ with a monoclonal rabbit anti-cleaved-caspase- 3 primary antibody (Cell Signaling Technologies, Beverly, MA) at a dilution of 1:200, biotinylated secondary antibody, streptavidin peroxidase reagent, and 3, 3'-Diaminobenzidine. Finally, the sections were lightly counterstained with hematoxylin and observed under a light microscope (Olympus: $\times 400$ ).

\section{Quantitative RT-PCR analysis}

Total RNA was extracted from the kidney tissues using Trizol reagent (Invitrogen Carlsbad, CA, USA). The total RNA concentration was measured at $260 \mathrm{~nm}\left(\mathrm{OD}_{260}\right)$. Pure RNA possessed an $\mathrm{OD}_{260} / \mathrm{OD}_{280}$ ratio of 1.6-1.9. The total RNA $(0.25 \mu \mathrm{g})$ was reverse-transcribed to cDNA using random primer and High-capacity cDNA reverse transcription kits (Applied Biosystems, Foster City, California, USA). Transcript expression was analyzed using quantitative polymerase chain reaction (qPCR) analysis with a Step One Plus ${ }^{\text {'m" }}$ (Applied Biosystems) using Taqman EXPRESS qPCR SuperMix Universal (Invitrogen). Values for each gene were normalized to the relative quantity of glyceraldehyde-3phosphate dehydrogenase (GAPDH) mRNA in each sample. The primers (Applied Biosystems) used for the PCR reaction are as follows: Assay ID; Bax; Rn02532082_g1, Assay ID; Bcl-2; Rn99999125_m1, and Assay ID; GAPDH; Rn99999916_s1. The relative concentration of products was calculated using the comparative $\mathrm{C}_{\mathrm{T}}\left(2^{-\Delta \Delta C \mathrm{~T}}\right)$ method, according to the instructions supplied by Applied Biosystems.

\section{Western blot analysis}

Total proteins were extracted from fresh frozen kidney tissues. The tissues were homogenized in $500 \mu \mathrm{l}$ of RIPA buffer. Homogenates were treated with protease inhibitors and centrifuged in a pre-chilled rotor at $15,000 \times \mathrm{g}$ for $15 \mathrm{~min}$. Supernatants were stored at $-70^{\circ} \mathrm{C}$. Protein content was measured using a BCA'" Protein Assay Kit (PIERCE, IL, USA). Protein equivalent to $40 \mu \mathrm{g}$ was separated on 10\% SDS-PAGE gel and transferred onto nitrocellulose membranes. Membranes were treated with Odyssey ${ }^{\circledR}$ blocking buffer followed by an overnight incubation at $4^{\circ} \mathrm{C}$ with primary antibody (rabbit anti-Bax, dilution 1:500; rabbit anti-Bcl-2, dilution 1:500; rabbit anti-cleaved caspase-3, dilution 1:500; rabbit anti-actin, dilution 1:2000; Cell Signaling Technology, Beverly, MA). Blots were then incubated with goat anti-rabbit LI-COR IRDye 680 antibody (dilution 1:5000). Densitometric analysis of bands was performed using LI-COR Odyssey ${ }^{\circledR}$.

\section{Statistical analysis}

Data were expressed as mean \pm SD. The means of the different groups were evaluated by one-way analysis of variance or unpaired t-test. The level of statistical significances was defined as $\mathrm{p}<0.05$.

\section{RESULTS}

PE extract has been used for the renoprotective effect of CI-AKI which was demonstrated by our work. A previous study has demonstrated that antioxidant property of PE extract accounts for the preventive effect in CI-AKI, which all rats in the CM group exhibited a significant increase in blood urea nitrogen (BUN) and $\mathrm{Cr}$ concentrations when compared to control. However, rats treated with PE before receiving $\mathrm{CM}$ had an improved renal function. Addition, histological examination of the kidney tissues showed extensive tubular damages in the CM group and also was markedly different from those in the control kidney. In contrast, rats pretreated with PE demonstrated a significantly decreased histopathological alternation of the kidney tissues when compared with the CM group [22].
In the present study, an investigation of the anti-apoptotic effects of PE extract to ameliorate in CI-AKI was performed. It was found that the expression levels of Bcl-2 and Bax were assessed. In the CM group, the expression of Bcl-2 mRNA was significantly downregulated while the expression of Bax mRNA was upregulated. However, when pre-treated with PE, there was a significantly reduced expression of Bax and increased expression of Bcl-2 levels, as shown in Fig. 1a. Moreover, a significant increase of Bax protein level and a significant decrease of Bcl-2 protein level, as indicated by Western blot analysis, were found in the kidney tissues from the CM rats compared with the control rats. Interestingly, the PE pre-treated CM rats appeared to have the augmented expression of $\mathrm{Bcl}-2$ and the reduced expression of Bax (Fig. 1b). Furthermore, Western blot analysis and IHC study revealed the expression of the apoptotic protein cleaved caspase- 3 protein in the renal tissue. This protein was increased in the CM group and was reduced in the CM + PE group (Fig. 2).

\section{DISCUSSION}

The present study has demonstrated the renoprotective effect of PE on CI-AKI in rats, which was mediated through suppression of apoptosis. We found that pre-treatment with PE at $500 \mathrm{mg} / \mathrm{kg} / \mathrm{day}$ for 5 days before the CI-AKI induction improved function and structure of renal tissues as well as markedly suppressed renal tubular injuries in the $\mathrm{CI}-$ AKI rats [22].

CI-AKI is a common clinical complication in patients receiving CM administration. The morbidity and mortality rates associated with AKI remain high, especially in the population at risks such as elderly, diabetes, and chronic kidney diseases [23]. Therefore, it is necessary to find an agent for preventing this serious complication. At present, there is no specific recommended therapy. Thus, the alternative therapy including nutritional intervention and supportive care which showed an improvement of the clinical outcomes of patients with AKI has been continuously encouraged [24]. The oxidative stress and apoptosis play important roles in the cellular pathogenesis of CI-AKI. The current body of evidence indicated that direct tubular toxicity of contrast agent, renal medullary ischemia, and generation of ROS leading to increased oxidative stress were responsible for the renal tubular cell apoptosis [8]. Therefore, many studies have focused on the identification of novel agents that can scavenge ROS and/or reduce apoptosis. Nowadays, several studies have discovered the antioxidant active constituents from natural plant extracts that could ameliorate CM-induced nephropathy in rats. These agents include magnolin [25], curcumin [7], and xuezhikang [26]. Recently, our previous study showed that pre-treatment with PE extract exhibited the antioxidant effect in CI-AKI rats as shown by the improvement of the oxidative-status parameters including lower renal malondialdehyde, higher renal Total antioxidant capacity (TAC), Superoxide dismutase (SOD) and catalase (CAT) activity, the improved renal function, and the attenuation of the severity of pathological damage [22]. However, its efficacy in preventing CI-AKI through anti-apoptotic has not been investigated.

P. emblica is widely used in Thai traditional medicine and Ayurvedic Medicine for the treatment of various diseases. The fruit of PE is known as a rich source of ascorbic acid [12,13] gallic acid [14], and also contains a mixture of phenolic compounds including flavonoids and tannins [15]. The major substrate which acts to be antioxidant in PE is gallic acid, Vitamin C, and others depending on the technique that separates the compound from PE $[27,28]$. In the present study, PE extract has been identified by high-performance liquid chromatography analysis. It was found that gallic acid was one of the main active constituents [22]. Because gallic acid is a polyphenolic compound with multiple hydroxyl groups which are able to donate its proton to break the chain reaction of free radicals [29,30], it was demonstrated as an excellent antioxidant with high free radical scavenging effect $[29,31]$. Moreover, gallic acid has been demonstrated to possess a wide range of pharmacological effects including antioxidant, anti-apoptosis, anticholestatic, anti-inflammatory, and immune-modulating activities as shown in nephroprotective, neuroprotective, and hepatoprotective animal models [32-34]. Furthermore, the renoprotective effect of 


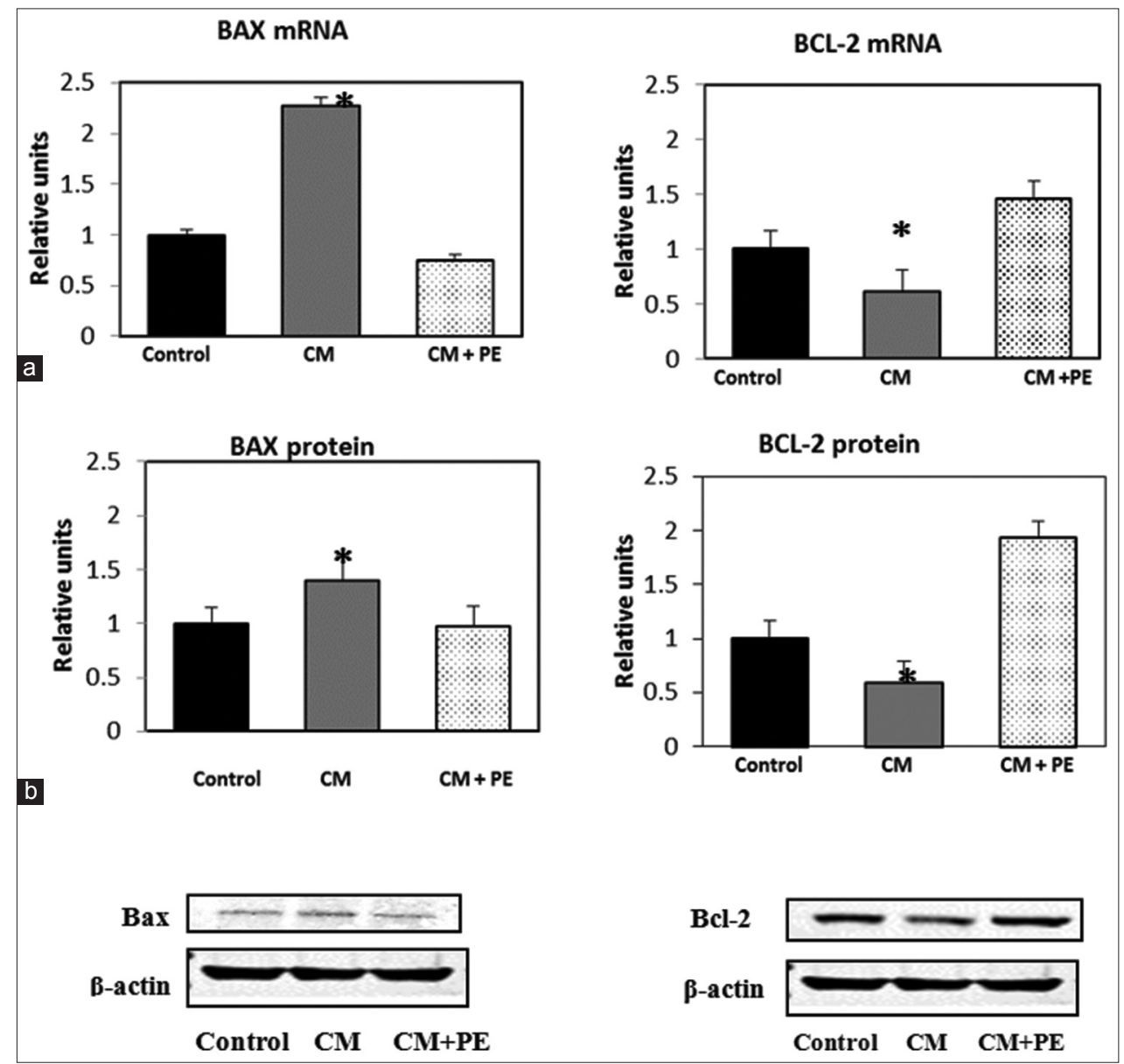

Fig. 1: Effect of PE on (a) the apoptotic gene and (b) protein expression in the study groups: ${ }^{* *} \mathrm{p}<0.05$; CM versus CM + PE

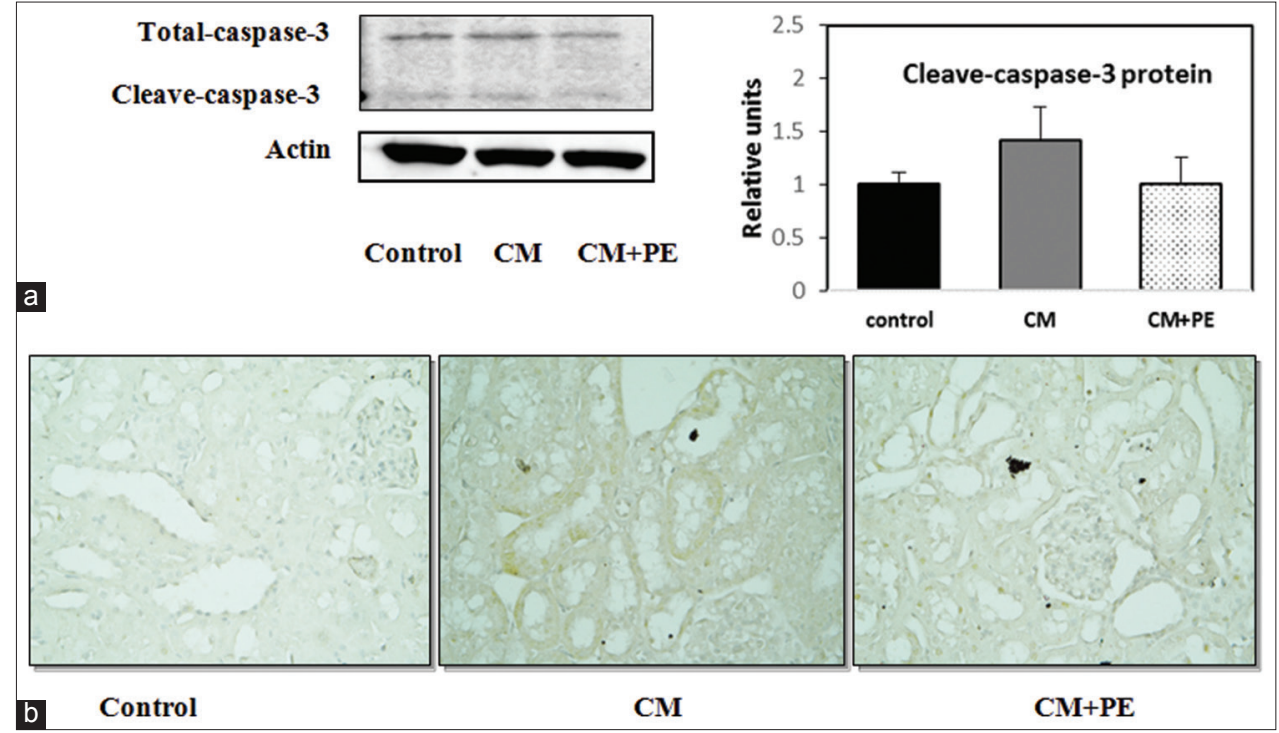

Fig. 2: Changes in cleaved caspase-3 activity in rat renal, Cleaved-caspase- 3 expression as assessed by Western blotting (a) and by immunostaining (b); alteration in the histological feature of renal tubules and glomerulus in the CM group with strong immunostaining of cleaved caspase $-3(\times 400)$

gallic acid from its antioxidant and anti-inflammation properties was demonstrated in many experimental studies in AKI [35].

The current study demonstrated that pre-administration of rats with PE extract ameliorated the deterioration of renal function and histopathological changes found in kidney injuries in CI-AKI rats. After pretreating rats with PE (CM + PE group), the BUN and creatinine levels significantly decreased compared with the CM group. Overall, our results suggested that PE extract could prevent renal damages and could help to preserve the kidney function. 
As apoptosis is one of the pathophysiological mechanisms of CIAKI, the anti-apoptotic property of PE extract against CM has been investigated [36,37]. It has been reported that both mitochondrial and intrinsic apoptosis pathways participated in the tubular injuries of Contrast induced nephropathy (CIN) [38]. Suppressing apoptosis pathways may attenuate pathological alterations in CIN. Since Bcl2 is an anti-apoptotic protein that can inhibit caspase-3, increased Bcl-2 levels and decreased caspase- 3 activity might contribute to reduced apoptosis [39]. As expected, rats in the CM group exhibited the upregulating expression of Bax mRNA and protein levels, the downregulating expression of Bcl-2 mRNA and protein, and increased level of cleaved caspase-3. Interestingly, pretreatment with PE extract decreased the Bax and cleaved caspase-3 while increased Bcl-2 level. Our findings were consistent with the evidence of anti-apoptotic effect on the nephroprotection that has been previously demonstrated in a study using E. officinalis against cisplatin-induced nephrotoxicity [40]. Moreover, the anti-apoptotic activity of gallic acid has been demonstrated to ameliorate an ischemia-reperfusion injury on gastric mucosa [41]. Overall, this study using PE extract which contains gallic acid as one of the active compounds has demonstrated the renoprotective effect, and we convincingly proposed that this effect was mediated by the antiapoptotic properties.

\section{CONCLUSION}

To our best knowledge, this study is the first in the literature demonstrating that the anti-apoptotic activities of PE extract could attenuate renal injury in the CI-AKI model. Thus, PE extract is a possible novel candidate for preventing kidney damages from the CM. This warrants an extensive exploration of its beneficial properties.

\section{ACKNOWLEDGMENTS}

This work was supported by Thammasat University Research Fund.

\section{COMPLIANCE WITH ETHICAL STANDARDS ETHICAL STATEMENT}

The experimental protocol was approved by the Animal Ethics Committee of Faculty of Medicine, Thammasat University. Registration No.AE $007 / 2015$

\section{DECLARATION OF CONFLICT OF INTEREST}

The authors declare that they have no conflicts of interest.

\section{REFERENCES}

1. Berns AS. Nephrotoxicity of contrast media. Kidney Int 1989;36:730-40.

2. Rudnick MR, Goldfarb S, Tumlin J. Contrast-induced nephropathy: Is the picture any clearer? Clin J Am Soc Nephrol 2008;3:261-2.

3. Gruberg L, Mintz GS, Mehran R, Gangas G, Lansky AJ, Kent KM, et al. The prognostic implications of further renal function deterioration within $48 \mathrm{~h}$ of interventional coronary procedures in patients with preexistent chronic renal insufficiency. J Am Coll Cardiol 2000;36:1542-8.

4. McCullough PA, Choi JP, Feghali GA, Schussler JM, Stoler RM, Vallabahn RC, et al. Contrast-induced acute kidney injury. J Am Coll Cardiol 2016;68:1465-73.

5. Aspelin P, Aubry P, Fransson SG, Strasser R, Willenbrock R, Berg KJ, et al. Nephrotoxic effects in high-risk patients undergoing angiography. N Engl J Med 2003;348:491-9.

6. Brezis M, Rosen S. Hypoxia of the renal medulla its implications for disease. N Engl J Med 1995;332:647-55.

7. Buyuklu M, Kandemir FM, Ozkaraca M, Set T, Bakirci EM, Topal E, et al. Protective effect of curcumin against contrast induced nephropathy in rat kidney: What is happening to oxidative stress, inflammation, autophagy and apoptosis? Eur Rev Med Pharmacol Sci 2014:18:461-70.

8. Quintavalle C, Brenca M, De Micco F, Fiore D, Romano S, Romano MF, et al. In vivo and in vitro assessment of pathways involved in contrast media-induced renal cells apoptosis. Cell Death Dis 2011;2:e155.

9. Mueller C, Buerkle G, Buettner HJ, Petersen J, Perruchoud AP, Eriksson U, et al. Prevention of contrast media-associated nephropathy: Randomized comparison of 2 hydration regimens in 1620 patients undergoing coronary angioplasty. Arch Intern Med 2002;162:329-36.
10. Spargias K, Alexopoulos E, Kyrzopoulos S, Iokovis P, Greenwood DC, Manginas A, et al. Ascorbic acid prevents contrast-mediated nephropathy in patients with renal dysfunction undergoing coronary angiography or intervention. Circulation 2004;110:2837-42.

11. Li Y, Liu Y, Fu L, Mei C, Dai B. Efficacy of short-term high-dose statin in preventing contrast-induced nephropathy: A meta-analysis of seven randomized controlled trials. PLoS One 2012;7:e34450.

12. Tarwadi K, Agte V. Antioxidant and micronutrient potential of common fruits available in the Indian subcontinent. Int J Food Sci Nutr 2007;58:341-9.

13. Krishnaveni M, Mirunalini S. Therapeutic potential of Phyllanthus emblica (amla): The ayurvedic wonder. J Basic Clin Physiol Pharmacol 2010;21:93-105.

14. Kumaran A, Karunakaran RJ. Nitric oxide radical scavenging active components from Phyllanthus emblica L. Plant Foods Hum Nutr 2006;61:1-5.

15. Zhang YJ, Tanaka T, Iwamoto Y, Yang CR, Kouno I. Novel norsesquiterpenoids from the roots of Phyllanthus emblica. J Nat Prod 2000;63:1507-10.

16. Ngamkitidechakul C, Jaijoy K, Hansakul P, Soonthornchareonnon N, Sireeratawong S. Antitumour effects of Phyllanthus emblica L.: Induction of cancer cell apoptosis and inhibition of in vivo tumour promotion and in vitro invasion of human cancer cells. Phytother Res 2010;24:1405-13.

17. Baliga MS, Dsouza JJ. Amla (Emblica officinalis Gaertn), a wonder berry in the treatment and prevention of cancer. Eur J Cancer Prev 2011;20:225-39

18. Ihantola-Vormisto A, Summanen J, Kankaanranta H, Vuorela H, Asmawi ZM, Moilanen E, et al. Anti-inflammatory activity of extracts from leaves of Phyllanthus emblica. Planta Med 1997;63:518-24.

19. Bandyopadhyay SK, Pakrashi SC, Pakrashi A. The role of antioxidant activity of Phyllanthus emblica fruits on prevention from indomethacin induced gastric ulcer. J Ethnopharmacol 2000;70:171-6.

20. Kusirisin W, Srichairatanakool S, Lerttrakarnnon P, Lailerd N, Suttajit M, Jaikang C, et al. Antioxidative activity, polyphenolic content and anti-glycation effect of some thai medicinal plants traditionally used in diabetic patients. Med Chem 2009;5:139-47.

21. Guo X, Ni J, Liu X, Xue J, Wang X. Phyllanthus emblica L. Fruit extract induces chromosomal instability and suppresses necrosis in human colon cancer cells. Int J Vitam Nutr Res 2013;83:271-80.

22. Tasanarong A, Kongkham S, Itharat A. Antioxidant effect of Phyllanthus emblica extract prevents contrast-induced acute kidney injury. BMC Complement Altern Med 2014;14:138.

23. Ueda N, Kaushal GP, Shah SV. Apoptotic mechanisms in acute renal failure. Am J Med 2000;108:403-15.

24. Yaklin KM. Acute kidney injury: An overview of pathophysiology and treatments. Nephrol Nurs J 2011;38:13-8.

25. Wang F, Zhang G, Zhou Y, Gui D, Li J, Xing T, et al. Magnolin protects against contrast-induced nephropathy in rats via antioxidation and antiapoptosis. Oxid Med Cell Longev 2014;2014:203458.

26. Chu S, Hu L, Wang X, Sun S, Zhang T, Sun Z, et al. Xuezhikang ameliorates contrast media-induced nephropathy in rats via suppression of oxidative stress, inflammatory responses and apoptosis. Ren Fail 2016;38:1717-25.

27. Scartezzini P, Speroni E. Review on some plants of Indian traditional medicine with antioxidant activity. J Ethnopharmacol 2000;71:23-43.

28. Habib-ur-Rehman, Yasin KA, Choudhary MA, Khaliq N, Atta-urRahman, Choudhary MI, et al. Studies on the chemical constituents of Phyllanthus emblica. Nat Prod Res 2007;21:775-81.

29. Lu Z, Nie G, Belton PS, Tang H, Zhao B. Structure-activity relationship analysis of antioxidant ability and neuroprotective effect of gallic acid derivatives. Neurochem Int 2006;48:263-74.

30. Stupans I, Kirlich A, Tuck KL, Hayball PJ. Comparison of radical scavenging effect, inhibition of microsomal oxygen free radical generation, and serum lipoprotein oxidation of several natural antioxidants. J Agric Food Chem 2002;50:2464-9

31. van Acker SA, van den Berg DJ, Tromp MN, Griffioen DH, van Bennekom WP, van der Vijgh WJ, et al. Structural aspects of antioxidant activity of flavonoids. Free Radic Biol Med 1996;20:331-42.

32. Priscilla DH, Prince PS. Cardioprotective effect of gallic acid on cardiac troponin-T, cardiac marker enzymes, lipid peroxidation products and antioxidants in experimentally induced myocardial infarction in Wistar rats. Chem Biol Interact 2009;179:118-24.

33. Ban JY, Nguyen HT, Lee HJ, Cho SO, Ju HS, Kim JY, et al. Neuroprotective properties of gallic acid from sanguisorbae radix on amyloid beta protein (25--35)-induced toxicity in cultured rat cortical neurons. Biol Pharm Bull 2008;31:149-53. 
34. Rasool MK, Sabina EP, Ramya SR, Preety P, Patel S, Mandal N, et al. Hepatoprotective and antioxidant effects of gallic acid in paracetamolinduced liver damage in mice. J Pharm Pharmacol 2010;62:638-43.

35. Prasad L, Khan TH, Jahangir T, Sultana S. Effect of gallic acid on renal biochemical alterations in male Wistar rats induced by ferric nitriloacetic acid. Hum Exp Toxicol 2006;25:523-9.

36. Caiazza A, Russo L, Sabbatini M, Russo D. Hemodynamic and tubular changes induced by contrast media. Biomed Res Int 2014;2014:578974.

37. Linkermann A, De Zen F, Weinberg J, Kunzendorf U, Krautwald S. Programmed necrosis in acute kidney injury. Nephrol Dial Transplant 2012;27:3412-9.

38. Romano G, Briguori C, Quintavalle C, Zanca C, Rivera NV, Colombo A, et al. Contrast agents and renal cell apoptosis. Eur Heart J
2008;29:2569-76

39. Goodman AI, Olszanecki R, Yang LM, Quan S, Li M, Omura $\mathrm{S}$, et al. Heme oxygenase-1 protects against radiocontrastinduced acute kidney injury by regulating anti-apoptotic proteins. Kidney Int 2007;72:945-53.

40. Malik S, Suchal K, Bhatia J, Khan SI, Vasisth S, Tomar A, et al. Therapeutic potential and molecular mechanisms of Emblica officinalis Gaertn in countering nephrotoxicity in rats induced by the chemotherapeutic agent cisplatin. Front Pharmacol 2016;7:350.

41. Mard SA, Mojadami S, Farbood Y, Gharib Naseri MK. The antiinflammatory and anti-apoptotic effects of gallic acid against mucosal inflammation and erosions-induced by gastric Ischemia-reperfusion in rats. Vet Res Forum 2015;6:305-11. 